\title{
PATRONES DE FORMULACIÓN DE OBJETIVOS EN ARTÍCULOS DE INVESTIGACIÓN EN LAS DISCIPLINAS DE LINGÜÍSTICA Y BIOTECNOLOGÍA ESCRITOS EN INGLÉS*
}

\author{
Violeta Cautín-Epifani* \\ Pontificia Universidad Católica de Valparaíso \\ Instituto de Literatura y Ciencias del Lenguage \\ Valparaíso, Chile
}

\author{
Jadranka Gladic Miralles ${ }^{\star \star \star}$ \\ Pontificia Universidad Católica de Chile \\ Facultad de Letras \\ Santiago, Chile
}

\begin{abstract}
Resumen: El presente trabajo, enmarcado en el área de la investigación de la construcción y transmisión del conocimiento a través del discurso académico, indaga acerca de los patrones funcionales y textuales de formulación de objetivos en un corpus de 20 artículos de investigación (AI) de las disciplinas de Lingüística y Biotecnología, escritos en idioma inglés. Los resultados generales indican que existe similitud entre los cinco patrones identificados por González (2011) y los encontrados en la presente investigación con textos escritos en inglés. Igualmente, se detectan diferencias en el uso de estos patrones en las disciplinas estudiadas en esta investigación. Finalmente, nuestro estudio arroja un nuеvo patrón funcional y textual de formulación de objetivos en AI el que se relaciona con la presentación del objetivo de investigación a través de la metodología utilizada.
\end{abstract}

Palabras clave: Artículo de investigación. Resumen. Patrones. Discurso académico. Discurso disciplinar.

1 INTRODUCCIÓN

Entendemos que el discurso académico disciplinar refiere a las construcciones discursivas que se producen y circulan en contextos académicos, a partir de estilos propios de cada comunidad ${ }^{1}$ de práctica inmersa en una disciplina (HYLAND, 2002). En general,

\footnotetext{
* Este artículo fue posible gracias al apoyo del Programa de Formación de Capital Humano de CONICYT 21100900 y 22110119 . Una versión preliminar de esta investigación se presentó en ALED Chile (2012), la que además contó con la participación de Ariel Mendoza Ríos y Carolina Aguilera Araya.

** E-mail: violeta.cautin@gmail.com

****E-mail: jagladic@uc.cl

${ }^{1}$ Por comunidad entendemos: "constructo sociocognitivo, artefacto cultural -regulado por pautas y normas convenidas, que generan derechos y obligaciones- conformado por un número variable de sujetos que interactúan -sin necesidad de copresencia física- de forma sistemática (acordando o discrepando), a través del empleo recurrente de géneros discursivos diversos - producidos en diferentes modos y soportes, por medio del uso intencional o intuitivo de estrategias de interacción-, y en cuyo seno se adquieren y desarrollan competencias, se asumen roles y papeles comunicativos, se instauran y consolidan lazos de algún tipo y se vehiculan representaciones sociales" (SAL PAZ; MALDONADO, 2013, en VELA DELFA, 2016, p. 56).
} 
cada comunidad disciplinar se conforma a partir del uso de prácticas lectoras y escritoras particulares (RUSSELL, 1997; CASSANY; MORALES, 2008; PARODI, 2010, 2015), las que reflejan y constituyen sus propias prácticas sociales, y, por lo tanto, ponen de manifiesto las maneras en que las comunidades gestionan su conocimiento (IBÁÑEZ; MONCADA; SANTANA, 2015). Estas prácticas discursivas son desarrolladas por el conjunto de sus miembros, de modo interrelacionado con el entorno físico, la cultura, la lengua, entre otros (HYLAND, 2016). En este contexto, el estudio de los géneros discursivos que se producen en una disciplina en particular resulta una manera provechosa de describir los diferentes modos en los que las comunidades disciplinares se constituyen discursivamente.

En el ámbito académico, uno de los géneros que ha atraído mayor atención es el artículo de investigación (AI). Así, podemos encontrar trabajos que lo han abordado desde distintas perspectivas, tales como la descripción de su estructuración retórica (SWALES, 1990, 2004; SABAJ; MATSUDA; FUENTES, 2010, SABAJ; TORO; FUENTES, 2011, KUHI; HAJI-HATAMLOO, 2016), la descripción de sus elementos metadiscursivos (ABDI; AHMADI, 2015; CAO; HU, 2014; HYLAND, 2005; McGRATH; KUTEEVA, 2012; MUR DUEÑAS, 2011), relaciones de coherencia (IBÁÑEZ; MONCADA; SANTANA, 2015), títulos (ENTRALGO; SALAGER-MEYER; LUZARDO, 2014), procedimientos de atribución de conocimiento (SABAJ; PÁEZ, 2010; BONILLA, 2015), patrones de progresión temática (FAN; ZHANG, 2016), entre otros.

En el presente trabajo nos enfocamos, particularmente, en indagar los patrones de formulación de objetivos en AI escritos en inglés en las disciplinas de Lingüística y Biotecnología $^{2}$. En este sentido, retomamos la línea investigativa iniciada por González (2011) para artículos escritos en español y expandimos su trabajo al idioma inglés.

Dar cuenta del objetivo de una investigación es parte importante del reporte de esta. De hecho, en general, los estudios que han indagado sobre la organización estructural de los AI, en diferentes disciplinas, coinciden en indicar la presentación de estos como un paso retórico obligatorio, bien dentro del resumen del AI o de su introducción (SWALES, 1990; BHATIA, 1994; DOS SANTOS, 1996; HYLAND, 2000; LORÉS, 2004; STOTESBURY, 2006; PHO, 2008; LI; KIRBI, 2014; PIQUÉNOGUERA; CAMAÑO-PUIG, 2015; MOHAMMADI, 2016). En este sentido, conocer los patrones de formulación de los objetivos fortalece el conocimiento sobre las características particulares de los AI y, por ende, de la disciplina que se está revisando.

En los siguientes apartados presentaremos los antecedentes teóricos de nuestra investigación, enfocándonos, especialmente, en reseñar lo que se entiende por discurso académico disciplinar y AI. Además, daremos cuenta de las características de los patrones de formulación de objetivos descritos por González (2011). Luego, presentaremos el marco metodológico y los resultados de nuestro trabajo, para cerrar con las conclusiones y discusión derivadas del análisis propuesto.

\footnotetext{
${ }^{2}$ Se seleccionaron estas disciplinas por ser las que más se asemejaban a las indagadas por González (2011), dentro del corpus con el que contábamos para trabajar. Para una descripción más detallada del corpus estudiado y del proyecto en el que se enmarca ver Parodi (2010), Parodi (2012) y Parodi (2015).
} 
El discurso académico disciplinar se utiliza en contextos académicos, con propósitos diversos e implica procedimientos y estilos propios de una comunidad discursiva (HYLAND, 2000). En este sentido, cada comunidad disciplinar se conforma a partir del uso de prácticas discursivas desarrolladas por el conjunto de sus miembros, de modo interrelacionado con el entorno físico, la cultura y la lengua (RUSSELL, 1997; CASSANY; MORALES, 2008; MARTÍNEZ, 2015; PARODI, 2010; VÁSQUEZROCCA; PARODI, 2015). Al respecto Ibáñez, Moncada y Santana (2015) señalan que:

\begin{abstract}
[el discurso académico] se constituye como un discurso que refleja las prácticas sociales que ocurren al interior de cada comunidad disciplinar, y manifiesta las formas de pensar de estas, revelando de este modo, las diferentes maneras en que tales comunidades diseminan sus ideas y construyen saberes.
\end{abstract}

Así, cuando un nuevo miembro de una comunidad discursiva se enfrenta inicialmente a prácticas propias de su disciplina requiere que demuestre comprensión de un lenguaje altamente especializado y sea capaz de construir significados específicos establecidos previamente por sus miembros. En otras palabras, requiere de un proceso de enculturación o inserción a la comunidad discursiva, el que no se presenta siempre de manera natural, sino que demanda un esfuerzo consciente por parte de este miembro novato y de apoyo por parte de los miembros más experimentados de la comunidad (SÁNCHEZ; MONTES, 2016). Ahora bien, la dificultad de apropiación de los géneros discursivos propios de su disciplina no desaparece automáticamente en el momento que los sujetos aumentan su especialización. Uno de los esfuerzos que incluso los estudiantes de posgrado e investigadores en ejercicio deben realizar es aprender a comunicar los resultados de sus investigaciones por medio del AI y de otros géneros propios de la disciplina. Para llevar a cabo esta transmisión y producción de nuevo conocimiento, deben adecuarse a las normas de su comunidad, pero también a las que proponen las revistas científicas en las que aspiran a publicar, proceso que, además, exige una preparación particular (FERRER, 2009; CHOIS; JARAMILLO, 2016).

\title{
3 EL ARTÍCULO DE INVESTIGACIÓN 3
}

Siguiendo a Venegas (2007, p. 413) el AI se puede definir como:

el texto escrito, generalmente publicado en una revista especializada, que tiene como finalidad informar a una comunidad científica los resultados de un trabajo de investigación realizado mediante la aplicación del método científico, según las características de cada disciplina de la ciencia.

\footnotetext{
${ }^{3}$ No ahondamos en la descripción retórica de este género debido a que esta ya ha sido abordada en extenso en numerosas publicaciones anteriores.
} 
Además, diversos investigadores coinciden en sostener que, paralelamente a la función informativa, el AI cumple un propósito altamente persuasivo en tanto busca "persuadir a una audiencia especializada acerca de la importancia de ciertos hallazgos, de la novedad de ciertas conclusiones teóricas o bien de la necesidad de adoptar tal o cual modelo o política pública, entre otras funciones" (PINA-STRANGER et al., 2013, p. 70). Este género discursivo, en general, se organiza en categorías superestructurales o apartados altamente convencionalizados, que incluyen: título, resumen, introducción, metodología, resultados, discusión y conclusiones (SWALES, 1990; VENEGAS, 2007; PINA-STRANGER et al., 2013).

Asimismo, este género se encuentra fuertemente validado por la comunidad académica de expertos como uno de los más importantes para la comunicación del conocimiento científico, pues vehicula el nuevo conocimiento de la disciplina. En este sentido, se considera como un género altamente especializado "con un conocimiento menos estable en tanto es conocimiento en construcción" (PINA-STRANGER et al., 2013, p. 74). Sin embargo, y pese a su transversalidad, esto no significa que los rasgos del AI sean homogéneos. Diferentes investigaciones, desde diversas perspectivas, han comprobado su variación disciplinar (FLOTTUM; KINN; DAHL, 2006; BRUCE, 2009; SABAJ; PÁEZ, 2010; DONESCH-JEZO, 2016; SALEHI; BIRIA, 2016).

Para nuestra investigación hemos elegido analizar los apartados resumen e introducción de los AI que componen nuestro corpus. Nos centramos en estos apartados debido a que en la mayoría de las descripciones retóricas de las que tenemos conocimiento, la expresión de los objetivos se sitúa como parte obligatoria de estas secciones (SWALES, 1990; BHATIA, 1994; DOS SANTOS, 1996; HYLAND, 2000; LORÉS, 2004; STOTESBURY, 2006; PHO, 2008; LI; KIRBI, 2014; PIQUÉNOGUERA; CAMAÑO-PUIG, 2015; MOHAMMADI, 2016). Cabe hacer notar la excepción que supone el artículo de Ibáñez et al. (2015), en la que no se identifica como relevante u obligatoria la movida retórica que tiene que ver con la presentación del objetivo o propósito de la investigación. Sobre este punto comentaremos en nuestra discusión.

\section{PATRONES DE FORMULACIÓN DE OBJETIVOS EN ARTÍCULOS DE INVESTIGACIÓN}

Entendemos los objetivos como una guía para el proceso de investigación, los que "se constituyen como el elemento central de este proceso [...] se espera que cualquier reporte de investigación considere los objetivos de investigación al momento de publicar los resultados" (GONZÁLEZ, 2011, p. 403). De este mismo modo, estos objetivos encauzan el proceso investigativo y proveen información importante para evaluar la pertinencia y/o interpretar los resultados de los que se da cuenta en un AI.

González (2011), investigó sobre la expresión de los objetivos en un corpus de AI escritos en español, y describió cinco patrones de formulación predominantes en cuatro disciplinas (Historia, Linguiística, Literatura y Biología). Estos patrones se describen en la tabla 1. 
Tabla 1 - Patrones de formulación de objetivos en Al en español (González, 2011)

\begin{tabular}{|c|c|c|}
\hline Denominación & Patrón de formulación & Ejemplos $^{4}$ \\
\hline $\begin{array}{l}\text { Patrón A: Explicita el objetivo de la } \\
\text { investigación }\end{array}$ & $\begin{array}{l}\text { El objetivo de esta investigación + } \\
\text { verbo ser en pasado + verbo en } \\
\text { infinitivo }\end{array}$ & $\begin{array}{l}\text { "El objetivo de este trabajo fue } \\
\text { analizar las propiedades } \\
\text { morfológicas y semánticas del } \\
\text { sufijo -ero dentro de las creaciones } \\
\text { neológicas" }\end{array}$ \\
\hline $\begin{array}{l}\text { Patrón B: Explicita el objetivo del } \\
\text { artículo }\end{array}$ & $\begin{array}{l}\text { El artículo tiene por objetivo + } \\
\text { verbo en infinitivo } \\
\text { El objetivo de este artículo es }+ \\
\text { verbo en infinitivo }\end{array}$ & $\begin{array}{l}\text { "Este trabajo tiene por objetivo } \\
\text { analizar el poemario La Hybris } \\
\text { (2007) de la escritora argentina } \\
\text { Alicia Genovese, considerando la } \\
\text { configuración del yo lírico y, } \\
\text { asimismo, el tipo de política } \\
\text { genérico-sexual que éste despliega" }\end{array}$ \\
\hline $\begin{array}{l}\text { Patrón C: Explicita el objetivo del } \\
\text { autor }\end{array}$ & $\begin{array}{l}\text { El investigador/autor tiene (verbo } \\
\text { presente o pasado) un objetivo } \\
\text { (verbo eninfinitivo) } \\
\text { Mi objetivo es + verbo en infinitivo }\end{array}$ & $\begin{array}{l}\text { "Mi intento es poner en perspectiva } \\
\text { dialógica escrituras que se han } \\
\text { potenciado, secretamente, desde } \\
\text { algún lugar imaginado, en las } \\
\text { complicidades para la alteración de } \\
\text { la producción cultural en nuestro } \\
\text { continente" }\end{array}$ \\
\hline $\begin{array}{l}\text { Patrón D: Señala lo que hace el } \\
\text { artículo }\end{array}$ & $\begin{array}{l}\text { El artículo + verba dicendi + } \\
\text { actividad investigativa } \\
\text { El artículo + verbo realizativo en } \\
\text { presente }\end{array}$ & $\begin{array}{l}\text { "El artículo describe el tratamiento } \\
\text { periodístico del diario El Mercurio } \\
\text { acerca del } 11 \text { de septiembre de } \\
\text { 1973. Esta fecha, considerada un } \\
\text { hito en la historia nacional, es } \\
\text { objeto de un tratamiento que del } \\
\text { tono positivo pasa a uno } \\
\text { predominantemente neutro" }\end{array}$ \\
\hline $\begin{array}{l}\text { Patrón E: Señala lo que se hace en } \\
\text { el documento }\end{array}$ & $\begin{array}{l}\text { En el artículo se hace (tercera } \\
\text { persona singular) con el propósito } \\
\text { de + verbo en infinitivo } \\
\text { En el artículo + primera persona + } \\
\text { actividades investigativas. }\end{array}$ & $\begin{array}{l}\text { "En este artículo se presenta el } \\
\text { resultado del análisis de las } \\
\text { propiedades morfológicas y } \\
\text { semánticas del sufijo -ero en } \\
\text { creaciones neológicas por sufijación } \\
\text { en las variedades del españolde } \\
\text { Argentina, Chile y Uruguay, como } \\
\text { una manera de verificar su } \\
\text { vitalidad" }\end{array}$ \\
\hline
\end{tabular}

En la presente investigación se observaron los patrones de formulación de objetivos en AI escritos inglés en dos disciplinas: Lingüística y Biotecnología. En este sentido, se compararon los patrones encontrados por González (2011) con los encontrados en esta investigación, para dar cuenta de la concordancia o divergencia entre la formulación de objetivos en este género discursivo en ambos idiomas. A continuación, se describe con mayor detalle los pormenores de esta investigación.

5 MARCO METODOLÓGICO

La investigación tuvo un carácter descriptivo y se llevó a cabo mediante procedimientos cualitativos y conteo de frecuencia. Se analizó un corpus de 20 artículos de investigación científica de las disciplinas de Lingüística y Biotecnología (10 artículos por cada disciplina) escritos en inglés. Los artículos seleccionados para el presente trabajo

\footnotetext{
${ }^{4}$ Los ejemplos fueron tomados de González (2011).
} 
se tomaron del sub-corpus de AI pertenecientes al Corpus PUCV-2010 ${ }^{5}$. La selección de los artículos a analizar se realizó de forma aleatoria, pero tomó en cuenta solo AI escritos en inglés por hablantes nativos de ese idioma.

La metodología de análisis se basó en la identificación de segmentos textuales que dieran cuenta directa o indirectamente del planteamiento de los objetivos por parte del o los autores en los apartados resumen e introducción correspondientes a esos mismos AI.

El reconocimiento de que un segmento textual correspondía o no a la expresión de objetivos se realizó mediante el análisis de tres investigadores. Cada uno de ellos revisó y clasificó por separado cada segmento textual. En las situaciones donde hubo discrepancias, estas se desambiguaron mediante consenso por parte del grupo investigador. Así, se elaboraron colectivamente los análisis de frecuencia de ocurrencias de patrones, lo que permitió clasificar las diversas maneras de incluir los objetivos en las secciones introducción y resumen. Posteriormente, se procedió al levantamiento de una categoría nueva de clasificación de patrones de formulación de objetivos, la que será descrita en el apartado que sigue.

Finalmente, estos datos se compararon con los resultados de González (2011) con la finalidad de observar las posibles relaciones entre los patrones de formulación de objetivos en los AI de las disciplinas estudiadas y la incidencia que podría tener el idioma de producción de estos textos.

\section{RESULTADOS Y DISCUSIÓN}

Como ya se ha mencionado, para esta investigación se identificaron los objetivos presentados en los apartados resumen e introducción de 20 AI de las disciplinas de Lingüística y Biotecnología. Posteriormente, se analizaron los objetivos de modo de identificar sus patrones de formulación, los que se cuantificaron y clasificaron en relación con las diferentes disciplinas y apartados investigados.

En total contabilizamos 40 segmentos textuales identificados como objetivos, de los que el $45 \%$ corresponde a objetivos en AI de Biotecnología y 55\% corresponde a objetivos en AI de Lingüística. En relación con el apartado de aparición, el 58\% de estos se encontró en el resumen y el $42 \%$ restante en la introducción.

Tabla 2 - Cantidad y porcentajes totales de objetivos identificados

\begin{tabular}{lcccccc}
\hline & \multicolumn{2}{c}{ Resumen } & \multicolumn{2}{c}{ Introducción } & \multicolumn{2}{c}{ Total objetivos } \\
\cline { 2 - 7 } & Cantidad & Porcentaje & Cantidad & Porcentaje & Cantidad & Porcentaje \\
\hline Lingüística & 8 & 47 & 14 & 61 & 22 & 55 \\
Biotecnología & 9 & 53 & 9 & 39 & 18 & 45 \\
\hline Total & 17 & 42 & 23 & 58 & 40 & 100 \\
\hline
\end{tabular}

\footnotetext{
${ }^{5}$ Para una descripción detallada del Corpus PUCV-2010 y el proyecto en el que se enmarca ver Parodi (2010), Parodi (2012) y Parodi (2015). 
Asimismo, se pudo observar que, en general, el segmento textual correspondiente al objetivo aparece tanto en el apartado resumen como en el apartado introducción. En este sentido, la expresión de los objetivos de investigación parece ser obligatoria para los AI de Lingüística y Biotecnología escritos en idioma inglés en los apartados estudiados. Esto concuerda con los análisis y descripciones que se han realizado de la estructura retórica de los AI en diferentes disciplinas, en los que consideran la expresión del objetivo o propósito investigativo como una movida o paso obligatorio, especialmente, en el apartado resumen (SWALES, 1990; BHATIA, 1994; DOS SANTOS, 1996; HYLAND, 2000; LORÉS, 2004; STOTESBURY, 2006; PHO, 2008; LI; KIRBI, 2014; PIQUÉNOGUERA; CAMAÑN-PUIG, 2015; MOHAMMADI, 2016).

Tabla 3 - Cantidad y patrón de objetivos por apartado en ambas disciplinas

\begin{tabular}{lll|lll}
\hline $\begin{array}{l}\text { Código AI } \\
\text { Biotenología }\end{array}$ & Resumen & Introducción & $\begin{array}{l}\text { Código AI } \\
\text { Lingüística }\end{array}$ & Resumen & Introducción \\
\hline Bio106 & $1(\mathrm{~F})$ & $1(\mathrm{~A})$ & Lin828 & 0 & $1(\mathrm{E})$ \\
Bio110 & 0 & $1(\mathrm{E})$ & Lin195 & $1(\mathrm{E})$ & $4(2 \mathrm{E}-2 \mathrm{~B})$ \\
Bio109 & $1(\mathrm{~F})$ & 0 & Lin270 & 0 & $2(\mathrm{C}-\mathrm{A})$ \\
Bio104 & $1(\mathrm{E})$ & $1(\mathrm{~F})$ & Lin674 & $1(\mathrm{~A})$ & $1(\mathrm{~A})$ \\
Bio111 & $1(\mathrm{~F})$ & $1(\mathrm{~A})$ & Lin703 & $1(\mathrm{E})$ & $1(\mathrm{E})$ \\
Bio107 & $1(\mathrm{~F})$ & $1(\mathrm{E})$ & Lin790 & $1(\mathrm{~F})$ & $1(\mathrm{E})$ \\
Bio108 & $1(\mathrm{~F})$ & $1(\mathrm{E})$ & Lin1019 & $1(\mathrm{~A})$ & $1(\mathrm{D})$ \\
Bio105 & $1(\mathrm{~F})$ & $1(\mathrm{~F})$ & Lin1020 & $1(\mathrm{~A})$ & $1(\mathrm{~A})$ \\
Bio103 & $1(\mathrm{~F})$ & $1(\mathrm{~F})$ & Lin1022 & $1(\mathrm{E})$ & $1(\mathrm{E})$ \\
Bio102 & $1(\mathrm{E})$ & $1(\mathrm{~A})$ & Lin115 & $1(\mathrm{~A})$ & $1(\mathrm{~A})$ \\
\hline
\end{tabular}

\subsection{FORMULACIÓN DE OBJETIVOS POR APARTADO}

Como se aprecia en la tabla 3, en general, los AI analizados explicitan un objetivo en su apartado resumen y uno en su apartado introducción, no obstante, estos no siempre concuerdan en su patrón de formulación. En trece de los AI analizados existe una diferencia entre el patrón presentado en el resumen y en la introducción y solo en siete AI existe concordancia en el patrón utilizado en ambos apartados.

Cabe destacar que los AI de Lingüística en dos ocasiones presentaron la formulación de objetivos en más de un segmento textual dentro del mismo apartado, y no necesariamente realizada por el mismo patrón de formulación. A continuación, se presenta un caso en el que se aprecian dos segmentos textuales identificados como objetivos en una misma introducción, los que no presentan el mismo patrón de formulación. El ejemplo (1.a) presenta un patrón de formulación C: Explicita el objetivo del autor, mientras que el ejemplo (1.b) presenta un patrón de formulación A: Explicita el objetivo de la investigación.

(1.a) Our goal was to examine wether readers simultaneously monitor more than one situational dimensions. (LIN270)

(1.b) Another goal of this investigation was to examine the comprehension of ecologically valid materials, rather than experimenter-generated materials. (LIN270) 
Con relación a la ocurrencia de los diferentes patrones de formulación, el análisis de los segmentos textuales que corresponden a objetivos en ambos apartados dio cuenta de la presencia de todos los patrones reseñados anteriormente, excepto el patrón $\mathbf{D}$. Adicionalmente, se encontró un nuevo tipo de patrón de formulación de objetivos. Este fue denominado PATRÓN F: Señala el objetivo a través de la metodología y será descrito más adelante.

Con respecto a los porcentajes de ocurrencia de los diferentes patrones de formulación de objetivos, en primer lugar se encuentra el patrón $\mathbf{E}$ con un $35 \%$ de ocurrencias totales, al que le siguen el patrón $\mathbf{F}$ con un $28 \%$ y el patrón A con un $27 \%$. Por su parte, los patrones con menos ocurrencias son el patrón B $(7 \%)$ y el patrón $\mathbf{C}$ (3\%). Tal como se mencionó en párrafos anteriores, no se encontró formulación de objetivos a través del patrón D (Ver Tabla 4). En este sentido, se puede apreciar una diferencia con los artículos en español en los que este patrón sí aparece, tal como reporta González (2011).

\section{Tabla 4 - Tipo de formulación, patrón que lo caracteriza y porcentaje de ocurrencia en el corpus}

\begin{tabular}{|c|c|c|c|}
\hline Tipo & Patrón de formulación & Porcentaje & Ejemplos \\
\hline $\begin{array}{l}\text { A: Explicita el } \\
\text { objetivo de la } \\
\text { investigación }\end{array}$ & $\begin{array}{l}\text {-El objetivo de esta } \\
\text { investigación + verbo ser + } \\
\text { verbo en infinitivo } \\
\text {-Esta investigación/estudio } \\
\text { +verbo realizativo(actividades } \\
\text { investigativas) }\end{array}$ & 27 & $\begin{array}{l}\text { The primary aim of the research reported } \\
\text { here is to locate the source of language } \\
\text { comprehension impairments in patients } \\
\text { diagnosed with Alzheimer's disease (AD). } \\
\text { (LIN1020) }\end{array}$ \\
\hline $\begin{array}{l}\text { B: Explicita el } \\
\text { objetivo del } \\
\text { artículo }\end{array}$ & $\begin{array}{l}\text {-El artículo tiene por } \\
\text { objetivo/meta + verbo en } \\
\text { infinitivo. } \\
\text {-El objetivo de este artículo es } \\
\text { + verbo en infinitivo }\end{array}$ & 7 & $\begin{array}{l}\text { One major goal of this article is to present a } \\
\text { constructionist theory that makes decisive } \\
\text { predictions about the knowledge-based } \\
\text { inferences that are generated on-line during } \\
\text { narrative comprehension. (LIN195) }\end{array}$ \\
\hline $\begin{array}{l}\text { C: Explicita el } \\
\text { objetivo del autor }\end{array}$ & $\begin{array}{l}\text {-Mi/nuestro objetivo es/fue + } \\
\text { verbo en infinitivo }\end{array}$ & 3 & $\begin{array}{l}\text { Our goal was to examine wether readers } \\
\text { simultaneously monitor more than one } \\
\text { situational dimensions. (LIN195) }\end{array}$ \\
\hline $\begin{array}{l}\text { D: Señala lo que } \\
\text { hace el artículo }\end{array}$ & No se encontró & - & No se encontró \\
\hline $\begin{array}{l}\text { E: Señala lo que } \\
\text { se hace en el } \\
\text { documento }\end{array}$ & $\begin{array}{l}\text {-En el artículo }+ \text { yo/nosotros }+ \\
\text { verbo realizativo (actividades } \\
\text { investigativas) }\end{array}$ & 35 & $\begin{array}{l}\text { In this article, we examine whether language } \\
\text { comprehension routinely involves the } \\
\text { activation of visual motor representation. } \\
\text { (LIN128) }\end{array}$ \\
\hline $\begin{array}{l}\text { F: Señala el } \\
\text { objetivo a través } \\
\text { de la metodología }\end{array}$ & $\begin{array}{l}\text {-En este estudio/trabajo }+ \\
\text { construcción en voz pasiva }+ \\
\text { to }+ \text { verbo en infinitivo }\end{array}$ & 28 & $\begin{array}{l}\text { In this work, quantitative proteomics was } \\
\text { employed to identify early key players } \\
\text { involved in B-cell dysfunction induced by } \\
\text { glucotoxicity. (BIO109) }\end{array}$ \\
\hline
\end{tabular}

CAUTÍN-EPIFANI, Violeta; MIRALLES, Jadranka Gladic. Patrones de formulación de objetivos en artículos de investigación en las disciplinas de lingüística y biotecnología escritos en inglés. Linguagem em (Dis)curso LemD, Tubarão, SC, v. 18, n. 1, p. 35-48, jan./abr. 2018. 


\subsection{LEVANTAMIENTO DE UN NUEVO PATRÓN DE FORMULACIÓN DE OBJETIVOS}

En relación con los diferentes patrones de formulación de objetivos, el único que no ha sido reconocido ni descrito en el trabajo de González (2011) es el patrón F. Este alcanza un $28 \%$ de ocurrencia respecto del total de segmentos textuales identificados en el corpus analizado. En el caso del subcorpus de Linguiística, de un total de 22 segmentos textuales identificados como objetivos, solo el $5 \%$ fueron formulados mediante el patrón F. En cambio, en el de Biotecnología el nuevo patrón alcanza un 55\% de un total de 18 segmentos textuales identificados. Esto establece una diferencia importante entre la formulación de objetivos en ambas disciplinas, ya que se aprecia que los artículos de Biotecnología utilizarían, preferentemente, la estrategia de señalar el objetivo de investigación de manera conjunta con la metodología de la misma. Es posible que esto se deba a que en esta disciplina lo novedoso del artículo corresponda a la metodología a utilizar con un propósito específico y por esta razón se destaque en conjunto con el objetivo. La utilización de este patrón, igualmente, se puede deber a las constricciones de espacio, principalmente, en la elaboración de los resúmenes. Es posible que los autores hayan visto la necesidad de buscar estrategias para solapar estos dos elementos en un mismo segmento textual. A este tipo de formulación se le denominó "Señala el objetivo a través de la metodología" y su estructura está determinada por una aseveración delocutiva que está guiada por el siguiente patrón:

In this (work/study) + construcción en voz pasiva + to

+ verbo en infinitivo + sintagma nominal

En los ejemplos (2) y (3) se puede apreciar que destaca el verbo en voz pasiva acompañado de un infinitivo, lo que permite el distanciamiento de la actividad de escribir el artículo o el distanciamiento con lo que sucede discursivamente en el documento. Por el contrario, lo que se enfatiza en este patrón son los procesos de la actividad científica equivalentes a la metodología de la investigación misma:

(2) In this work, quantitative proteomics was employed to identify early key players involved in B-cell dysfunction induced by glucotoxicity. (BIO109)

(3) In this study, iTRAQ and 2-D LC MALDI-MS/MS have been used to characterise protein expression changes in the S. nodorum gna 1 strain versus the SN15 wild-type. (BIO111)

En el apartado resumen, la formulación de objetivos mediante el patrón $\mathbf{F}$ equivale al $47 \%$ de un total de 17 segmentos textuales identificados, mientras que en el apartado introducción, de un total de 23 segmentos textuales identificados solo un 13\% corresponde a la formulación de objetivos que se enuncian a través de la metodología.

Se aprecia, así, que este patrón parece ser más propio del resumen, posiblemente, debido a que las constricciones de espacio de este hacen necesario buscar recursos para condensar en un mismo segmento textual la metodología y el objetivo de la investigación, o, tal como lo señalamos anteriormente, es posible que esto se deba a que lo novedoso del artículo sea la metodología a utilizar en un contexto dado y por esto se decide destacar en el resumen de manera conjunta con el propósito de la investigación. 
En una investigación que buscó identificar las movidas retóricas que componen los resúmenes de la disciplina de Biología en español, Ibáñez, Moncada y Santana (2015) identificaron dos movidas en las que parece suceder el mismo fenómeno que señalamos para los AI estudiados de la disciplina de Biotecnología: se presenta el propósito de la investigación en conjunto con el método aplicado. Resulta interesante observar que en su investigación estas movidas no aparecieron con una frecuencia suficiente como para considerarlas obligatorias. Teniendo en cuenta que la investigación señalada, al igual que la de González (2011), se basó en AI escritos en español, podemos suponer que esta sería una diferencia en la manera de textualizar el discurso académico en español e inglés. Otra explicación podría ser la naturaleza de las disciplinas en cuestión. Es posible que, debido a que Biotecnología es una disciplina aplicada, se enfatice más en las técnicas para lograr los objetivos, razón por la que el patrón de formulación $\mathbf{F}$ aparece como preferente en el subcorpus indagado en esta investigación.

\subsection{FORMULACIÓN DE OBJETIVOS POR DISCIPLINA}

La formulación de objetivos por cada disciplina estudiada es variada, puesto que cada una utiliza diferentes patrones para dar cuenta de la relación entre el artículo y los objetivos subyacentes a la actividad de escribir. En el caso de Lingüística, predomina el patrón $\mathbf{E}$ con un $41 \%$ de un total de 22 objetivos identificados en el sub-corpus, seguido del patrón A que alcanza un 36\%. Mientras que los patrones B (14\%), F (5\%) y C (4\%) no presentan mayor preponderancia. El patrón $\mathbf{D}$ fue el único que no se encontró en esta disciplina. Al comparar estos resultados con los de González (2011) para artículos de Lingüística en español encontramos que en ambas investigaciones el patrón de formulación $\mathbf{E}$ es el que predomina para esta disciplina. Es decir, tanto en español como en inglés en los apartados resumen e introducción en AI de Linguiística se tiende de manera preferente a expresar los objetivos señalando lo que se realiza en el documento. En este sentido, se aprecia una mayor conciencia por parte de los autores respecto del proceso discursivo por el que pasa su investigación en tanto enfatizan el documento como el lugar en el que se construye la investigación, lo que se marca, principalmente, con el uso de la preposición in (en para español, ver GONZÁLEZ, 2011), tal como se aprecia en los ejemplos 4 y 5 .

(4) In this article, we propose and test a model of how readers construct these microworlds, or situation models. (LIN1122)

(5) In this paper we study supervised and semi-supervised classifications of e-mails. (LIN703)

Si atendemos ahora a los AI de la disciplina de Biotecnología, la preponderancia mayor la obtuvo el patrón $\mathbf{F}$ con un 55\%, seguido del patrón $\mathbf{E}$ con un $28 \%$ y el patrón A con un 17\%. Cabe señalar que en esta disciplina no se aprecia formulación de objetivos a través de los patrones B, C y D. Esto quiere decir que, en el caso de Biotecnología, no se le otorga importancia a explicitar o señalar el objetivo del autor o del artículo, sino más bien se formulan los objetivos de acuerdo a su relación con la investigación, el documento y su metodología. 
Se aprecia una diferencia en la variedad de objetivos encontrados en cada disciplina. De acuerdo a los datos obtenidos en esta investigación, en los AI de Linguística se presenta una mayor variedad de formulación de objetivos por sobre los AI de Biotecnología que tienden a repetir los mismos tres patrones de formulación, tal como se aprecia en la Tabla 5.

\section{Tabla 5 - Tipo de patrón de formulación por disciplina en porcentaje}

\begin{tabular}{lrr}
\hline Patrón de formulación & Lingüística & Biotecnología \\
\hline Explicita el objetivo de la investigación & 36 & 17 \\
Explicita el objetivo del artículo & 14 & - \\
Explicita el objetivo del autor & 4 & - \\
Señala lo que hace el artículo & - & - \\
Señala lo que se hace en el documento & 41 & 28 \\
Señala el objetivo a través de la metodología & 5 & 55 \\
\hline
\end{tabular}

\section{CONCLUSIONES}

En el presente artículo se da cuenta de los resultados de un estudio que comparó la formulación de objetivos de investigación en un corpus de textos del género AI, escritos en inglés, de dos disciplinas: Lingüística y Biotecnología. Esta investigación tenía como propósito indagar con datos empíricos respecto de la formulación de objetivos en los AI en el idioma inglés, en comparación con estudios similares para el español (GONZÁLEZ, 2011).

En general, se evidencia que los patrones de formulación identificados por González (2011) se mantendrían para el idioma inglés, es decir, son independientes del idioma de producción del AI. Además, se ha identificado un nuevo patrón denominado Patrón F: señala el objetivo a través de la metodología, cuya ocurrencia predomina en los AI de Biotecnología. Esto da cuenta de una diferencia disciplinar en relación con los patrones de formulación de objetivos encontrados. Mientras que en Biotecnología predomina la ocurrencia del patrón $\mathbf{F}$, en Lingüística la tendencia es hacia la predominancia del patrón $\mathbf{E}$, en el que se propone el documento como el momento y lugar en el que se realiza la investigación. En este sentido, y tal como lo comentamos anteriormente, se aprecia una mayor conciencia de los autores del proceso discursivo por el que pasa su investigación en tanto enfatizan el documento como el lugar en el que se construye la investigación. Esto sería concordante con lo señalado por González (2011) para esta disciplina en AI escritos en español.

Además de la diferencia en el patrón de formulación de objetivos predominante, se ha encontrado que los AI de la disciplina de Lingüística presentan una mayor diversidad de uso de estos patrones. En este sentido, se encontraron 5 tipos de patrones de formulación de objetivos diferentes, mientras que en Biotecnología solo se encontraron 3 tipos, y uno de ellos (patrón F) con más del 50\% de predominancia. Esto daría cuenta de que Biotecnología es una disciplina que presenta mayor homogeneidad en sus prácticas discursivas. Es posible que los expertos en Biotecnología no se sientan tan confiados en sus habilidades discursivas por los que tenderían a seguir con mayor fidelidad los patrones 
y modelos preestablecidos, lo que no sucedería en Lingüística, disciplina en la que los escritores tienden a variar más en los recursos retóricos que utilizan para dar cuenta de los objetivos de investigación. Quedará para estudios posteriores, particularmente con datos de corte más etnográfico, confirmar las razones de las diferencias aquí propuestas.

\section{REFERENCIAS}

ABDI, R.; AHMADI, P. Signposting propositions: a study of interactive metadiscourse marking in the composition of RAs across sciences. RALs, v. 6, p. 5-17, 2015.

BHATIA, V. Analyzing genre: Language use in professional setting. Londres: Longman, 1994.

BONILLA, L. Funciones discursivas de la cita directa en la presentación e interpretación de los datos de investigación en artículos de antropología social. Lenguaje, v. 43, n. 2, p. 271-300, 2015.

BRUCE, I. Results sections in sociology and organic chemistry articles: A genre analysis. English for Specific Purposes, v. 28, p.105-124, 2009.

CAO, F.; HU, G. Interactive metadiscourse in research articles: A comparative study of paradigmatic and disciplinary influences. Journal of Pragmatics, v. 66, p. 15-31, 2014.

CASSANY, D.; MORALES, O. Leer y escribir en la universidad: Hacia la Lectura y la Escritura crítica de géneros científicos. Revista Memoralia, v.5, n. 2, p. 69-82, 2008.

CHOIS, P.; JARAMILLO, L. La investigación sobre la escritura en posgrado: estado del arte. Lenguaje, v. 44, n.2, p. 227-259, 2016.

DONESCH-JEZO, E. Cross-cultural variability of research article abstracts from differente discourse communities. Pantwo i Spoleczenstwo, v. 16, n. 3, p. 33-60, 2016.

DOS SANTOS, M. The textual organization of research paper abstracts in applied linguistics. Text \& Talk, v. 16, n. 4, p. 481-499, 1996.

ENTRALGO, J.; SALAGER-MEYER, F.; LUZARDO, M. Títulos de artículos de investigación científica escritos en inglés: un estudio interdisciplinario. Revista de la Escuela de Idiomas Modernos, v. 31, p. 75100,2014

FAN, J.; ZHANG, Y. A quantitative study on discourse coherence in linguistic research articles from the perspective of thematic progression patterns. International Journal of Liberal Arts and Social Science, vol. 4, n. 7, p. 61-74, 2016.

FERRER, M. La escritura científica: ¿un problema de formación o de información? Acimed, v. 20, n.5, p. $1-8,2009$.

FLOTTUM, K.; KINN, T.; DAHL, T. "We now reporto on...” versus “Le tus now see how...”. Author roles and interaction with readers in research articles. In: HYLAND, K.; BONDI, M. (Eds.). Academic discourse across disciplines. Bern: Peter Lang, 2006. p. 203-224.

GONZÁLEZ, C. La formulación de los objetivos en artículos de investigación científica en cuatro disciplinas: Historia, Lingüística, Literatura y Biología. Linguagem em (Dis)curso, v. 11, n. 2, p. 401 429, 2011.

HYLAND, K. Disciplinary discourses: Social interactions in academic writing. London: Longman, 2000. Authority and invisibility: Authorial identity in academic writing. Journal of Pragmatics, v. 34, n. 8, p. 1091-1112, 2002.

Stance and engagement: a model of interaction in academic discourse. Discourse Studies, v. 7, p. 173-192, 2005.

Teaching and Researching Writing. London: Routledge, 2016.

IBÁNEZ, R.; MONCADA, F.; SANTANA, A. Variación disciplinar en el discurso académico de la Biología y del Derecho: un estudio a partir de las relaciones de coherencia. Onomáizen, v. 32, p. 101-131, 2015.

KUHI, D.; HAJI-HATAMLOO, N. An Investigation of the Generic Features of Research Articles Published in the Bulletin of Iranian Mathematical Society. Journal of Applied Linguistics, v. 8, n. 16, p. 110-137, 2015. 
LI, M.; KIRBY, J. The effects breadth and depth of English reading. Applied Linguistics, v. 36, n. 5, p. 611-634, 2014.

LORÉS, R. On research article abstracts: From rhetorical structure to thematic organization. English for Specific Purposes, v. 23, n. 3, p. 280-302, 2004.

MARTÍNEZ, J. El género Tesis Doctoral de Historia y Física: Descripción y variación retórico-funcional. En Parodi, G.; Burdiles, G. (Eds.). Leer y escribir en contextos académicos y profesionales: Géneros, corpus y métodos. Santiago: Ariel, 2015. p. 113-151.

McGRATH, L.; KUTEEVA, M. Stance and engagement in pure mathematics research articles: linking discourse features to disciplinary practices. English for Specific Purposes, v. 31, p. 161-173, 2012.

MOHAMMADI, B. Exploring gender differences in cross-disciplinary discourse: Interactional metadiscourse markers in the discussion section of research articles, Language in India, v. 16, n. 12, p. 169-189, 2016.

MUR DUEÑAS, P. An intercultural analysis of metadiscourse features in research articles written in English and in Spanish. Journal of Pragmatics, v. 43, p. 3068-3079, 2011.

PARODI, G. Multisemiosis y lingüística de corpus: Artefactos (multi) semióticos en los textos de seis disciplinas en el corpus PUCV-2010. Revista de Lingüística Teórica y Aplicada, v. 48, n. 2, p. 33-70, 2010.

¿Qué se lee en los estudios doctorales?: Estudio empírico basado en géneros a través del discurso académico de seis disciplinas. RLA. Revista de Lingüística Teórica y Aplicada, v.50, n.2, p. 89-119, 2012.

Variation across university genres in seven disciplines: A corpus-based study on academic written Spanish. International Journal of Corpus Linguistics, v. 20, n. 4, p. 469-499, 2015.

PHO, P. Research article abstracts in applied linguistics and educational technology: A study of linguistic realizations of rhetorical structure and authorial stance. Discourse Studies, v.10, n. 2, p. 231-250, 2008.

PINA- STRANGER, A.; SABAJ, O.; TORO, P.; MATSUDA, K. Estrategias académicas de inserción científica: una propuesta metodológica para el estudio de las reivindicaciones epistémicas en los artículos de investigación. Innovar, v. 23, n. 48, p. 67-82, 2013.

PIQUÉ-NOGUERA, C.; CAMAÑN-PUIG, R. El resumen del artículo de investigación: Análisis del género en un corpus de textos de Enfermería. Revista Signos, v. 48, n. 87, p. 77-94, 2015.

RUSSELL, D. Rethinking genre in school and society an activity theory analysis. Written communication, v.14, n. 4, p. 504-554, 1997.

SABAJ, O.; MATSUDA, K.; FUENTES, M. Un Modelo para la Homogeneización de las Clases Textuales de la Biblioteca Electrónica Scielo-Chile: la Variabilidad del Artículo de Investigación en Diversas Disciplinas. Información Tecnológica, v. 21, n. 6, p. 133-148, 2010.

SABAJ, O.; PÁEZ, D. Tipos y funciones de las citas en artículos de investigación de tres disciplinas. Literatura y Lingüística, v. 22, p. 117-134, 2011.

SABAJ, O.; TORO, P.; FUENTES, M. Construcción de un modelo de Movidas Retóricas para el análisis de Artículos de Investigación en español. Revista Onomázein, v. 24, n. 2, p. 245-271, 2011.

SAL PAZ, J.; MALDONADO, S. Delimitación y alcances de la voz comunidad en el marco de los estudios del discurso. Forma y Función, v. 26, n. 11, p. 111-140, 2013.

SALEHI, B.; BIRIA, R. Exploring Gender Differences in Cross-disciplinary Discourse: Interactional Metadiscourse Markers in the Discussion Section of Research Articles. Language in India, v. 16, p. 169$186,2016$.

SÁNCHEZ, V.; MONTES, S. El Programa de Lectura y Escritura Académicas de la Pontificia Universidad Católica de Chile: sus aportes para la inserción académica de los estudiantes. In: NATALE, L.; STAGNARO, D. (Comp.). Alfabetización académica: un camino hacia la inclusión en el nivel superior. Buenos Aires: Ediciones UNGS, 2016. p. 73-101.

STOTESBURY, H. Gaps and False Conclusions: Criticism in Research Article Abstracts across the Disciplines. In HYLAND, K.; BONDI, M. (Eds.). Academic Discourse Across Disciplines. New York: Peter Lang, 2006. p. 123-148.

SWALES, J. Genre analysis: English in academic and research settings. Cambridge: Cambridge University Press, 1990.

Research genres: Explorations and applications. Cambridge: Cambridge University Press, 2004.

CAUTÍN-EPIFANI, Violeta; MIRALLES, Jadranka Gladic. Patrones de formulación de objetivos en artículos de investigación en las disciplinas de lingüística y biotecnología escritos en inglés. Linguagem em (Dis)curso LemD, Tubarão, SC, v. 18, n. 1, p. 35-48, jan./abr. 2018. 
VÁSQUEZ-ROCCA, L.; PARODI, G. Relaciones retóricas y multimodalidad en un género 'importado': El Informe de Política Monetaria del discurso académico de la Economía. Calidoscópio, v. 13, n. 3, p. 388-405, 2015.

VELA DELFA, C. Una aproximación desde el correo electrónico desde una perspectiva diacrónica: evolución y asentamiento de un género discursivo. Cadernos de Linguagem e Sociedade, v.17, n. 2, p. 55$78,2016$.

VENEGAS, R. Clasificación de textos académicos en función de su contenido léxico-semántico. Revista Signos, v. 40, n. 63, p. 239-271, 2007.

\section{AGRADECIMIENTOS}

Las autoras agradecen la invaluable colaboración técnica de Ariel Mendoza Ríos (Pontificia Universidad Católica de Valparaíso) y Carolina Aguilera Araya (Pontificia Universidad Católica de Valparaíso), quienes, además, participaron en una versión preeliminar de esta investigación presentada en ALED (2012).

Recebido em: 29/03/17. Aprovado em: 21/01/18.

Title: Patterns of formulation of objectives of research articles written in English in two disciplines: linguistics and biotechnology

Authors: Violeta Cautín-Epifani; Jadranka Gladic Miralles.

Abstract: The present work, framed in the area of research on the construction and transmission of knowledge through academic discourse, inquiries about functional and textual patterns of goal formulation in a corpus of 20 research articles $(R A)$ of the subject of Linguistics and Biotechnology, written in English. The general results indicate that there is similarity between the five patterns identified by Gonzalez (2011) and those found in the present research with texts written in English. Similarly, differences in the use of these patterns are detected in the subjects studied in this research. Finally, the study reveals a new functional and textual pattern of formulation of objectives in $R A$ related to the presentation of the research objective through the methodology used.

Keywords: Research article. Abstract. Patterns. Academic discourse. Discourse of subject.

Título: Padrões de formulação de objetivos em artigos de investigação nas disciplinas de Linguística e Biotecnologia escritos em inglês

Autoras: Violeta Cautín-Epifani; Jadranka Gladic Miralles.

Resumo: O presente trabalho, emoldurado na área de investigação da construção e transmissão do conhecimento por meio do discurso acadêmico, indaga sobre os padrões funcionais e textuais de formulação de objetivos em um corpus de 20 artigos de investigação (AI) das disciplinas de Linguística e Biotecnologia, escritos em inglês. Os resultados gerais indicam que existe similaridade entre os cinco padrões identificados por González (2011) $e$ os encontrados na presente investigação com textos escritos en inglês. Igualmente detecta diferenças no uso desses padrões nas disciplinas estudadas nesta investigação. Finalmente, o estudo lança um novo padrão funcional e textual de formulação de objetivos en AI, que se relaciona com a apresentação do objetivo de pesquisa por meio da metodologia utilizada.

Palavras-chave: Artigo. Resumo. Padrões. Discurso acadêmico. Discurso de disciplina.

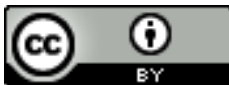

Este texto está licenciado com uma Licença Creative Commons Atribuição 4.0 Internacional.

CAUTÍN-EPIFANI, Violeta; MIRALLES, Jadranka Gladic. Patrones de formulación de objetivos en artículos de investigación en las disciplinas de lingüística y biotecnología escritos en inglés. Linguagem em (Dis)curso LemD, Tubarão, SC, v. 18, n. 1, p. 35-48, jan./abr. 2018. 